\title{
ASYMPTOTIC VARIANCE EXPRESSIONS FOR CLOSED-LOOP IDENTIFICATION AND THEIR RELEVANCE IN IDENTIFICATION FOR CONTROL
}

\author{
Michel Gevers ${ }^{\ddagger}, 1$, Lennart Ljung ${ }^{\S}$ and Paul M.J. Van den Hof H $^{\sharp, 2}$ \\ ${ }^{\ddagger}$ CESAME, Bâtiment Euler, Louvain University, B-1348 Louvain-la-Neuve, \\ Belgium. \\ , ${ }^{\S}$ Department of Electrical Engineering, Linköping University, S-581 83 Linköping, \\ Sweden. \\ * Mechanical Engineering Systems and Control Group, Delft University of \\ Technology, Mekelweg 2, 2628 CD Delft, The Netherlands.
}

\begin{abstract}
Asymptotic variance expressions are analysed for models that are identified on the basis of closed-loop data. The considered methods comprise the classical 'direct' and 'indirect' method, as well as the more recently developed indirect methods, employing coprime factorized models and model parametrizations based on the dual Youla/Kucera parametrization. The variance expressions are compared with the open-loop situation, and evaluated in terms of their relevance for subsequent modelbased control design. Additionally it is specified what is the optimal experimental situation in identification (open-loop or closed-loop), in view of the variance of the resulting model-based controller.
\end{abstract}

Keywords. System identification; closed-loop identification; asymptotic variance expressions, prediction error methods; model-based control design.

\section{INTRODUCTION}

When identifying dynamic models for the specific purpose of subsequent model-based control design it is argued that a closed-loop experimental setup during the identification experiments supports the construction of an identified model that is particularly accurate in that frequency region that is relevant for the control design. This mechanism which plays a major role in many contributions in the area of "identification for control", has been motivated mainly on the basis of bias considerations in the form of a "control-relevant" distribution of the bias over frequency (Gevers, 1993; Van den Hof and

\footnotetext{
1 M. Gevers acknowledges the financial support of the Belgian Programme on Interuniversitary Poles of Attraction.

2 Author to whom correspondence should be addressed.

E-mail: p.m.j.vandenhof@wbmt.tudelft.nl.
}

Schrama, 1995). Recently it has been shown in Hjalmarsson et al. (1996), that for a particular class of control design methods, also from a variance point of view closed-loop experiments are preferred over open-loop ones. In this paper we will first present the asymptotic variance expressions for identified models based on several different closed-loop identification methods, including the recently introduced indirect methods using a coprime factor model representation (Van den Hof et al., 1995) and the method employing a so-called dual Youla/ Kucera parametrization (Hansen and Franklin, 1988). The results for the classical 'direct' method (Ljung, 1993) are extended to also include variance expressions for the estimated noise model, while they are shown to remain the same for the mentioned alternative indirect methods. Consequences are shown for the variance of resulting model-based controllers for several types of controller designs. 


\section{PRELIMINARIES}

We will consider the closed-loop configuration as depicted in Fig. 1, where $G_{0}$ and $C$ are linear time-invariant, possibly unstable, finite dimensional systems, with $G_{0}$ strictly proper, while $C$ is a stabilizing controller for $G_{0} ; e$ is a white noise process with variance $\lambda_{0}$, and $H_{0}$ a stable and stably invertible monic transfer function. Signals $r_{1}$ and $r_{2}$ are external reference signals that are possibly available from measurements. For purpose of efficient notation, we will often deal with the signal

$$
r(t):=r_{1}(t)+C(q) r_{2}(t)
$$

being the result of external excitation through either $r_{1}$ or $r_{2}$. Additionally we will denote: $u(t)=u^{r}(t)+u^{e}(t)$

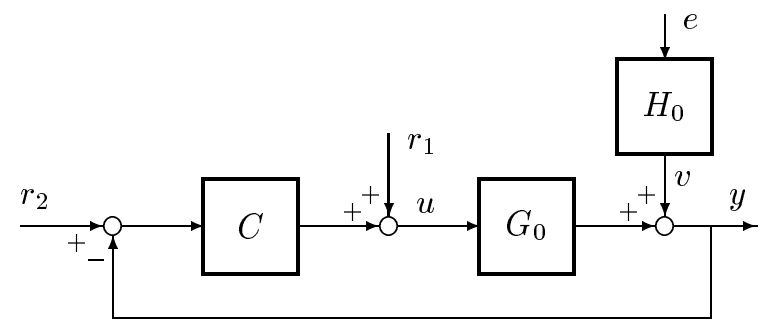

Fig. 1. Closed-loop configuration.

with

$$
\begin{aligned}
& u^{r}(t):=S_{0}(q) r(t), \\
& u^{e}(t):=-C(q) S_{0}(q) H_{0}(q) e(t),
\end{aligned}
$$

where the sensitivity function $S_{0}$ is given by $S_{0}(q):=$ $\left[1+C(q) G_{0}(q)\right]^{-1}$. For the corresponding spectra it follows that $\Phi_{u}=\Phi_{u}^{r}+\Phi_{u}^{e}$ with

$$
\Phi_{u}^{r}=\left|S_{0}\right|^{2} \Phi_{r} \quad \text { and } \Phi_{u}^{e}=\left|C S_{0}\right|^{2} \Phi_{v} .
$$

The arguments $q$ and $e^{i \omega}$ will be omitted when appropriate. We will consider parametrized models $G(q, \theta)$ for $G_{0}$ and $H(q, \theta)$ for $H_{0}$ with $\theta \in \Theta$, and we will use expressions $\mathcal{S} \in \mathcal{M}$ and $G_{0} \in \mathcal{G}$ to indicate the situations that both $G_{0}$ and $H_{0}$ or only $G_{0}$ can be modelled exactly within the model set. The variance expressions that are considered in this paper are asymptotic in both $n$ (model order) and $N$ (number of data), while $n / N$ is supposed to tend to 0 , as in the standard framework of Ljung (1987).

\section{DIRECT IDENTIFICATION}

The direct method of closed-loop identification is characterized by $\hat{\theta}_{N}=\arg \min _{\theta} \frac{1}{N} \sum_{t=0}^{N-1} \varepsilon(t, \theta)^{2}$ with

$$
\varepsilon(t, \theta)=H(q, \theta)^{-1}[y(t)-G(q, \theta) u(t)] .
$$

An expression for the asymptotic variance of the transfer function estimate can be given for the situation that $\mathcal{S} \in$
$\mathcal{M}$, and both plant model and noise are estimated. In this case (Ljung, 1987):

$$
\operatorname{cov}\left(\begin{array}{c}
\hat{G}\left(e^{i \omega}\right) \\
\hat{H}\left(e^{i \omega}\right)
\end{array}\right) \sim \frac{n}{N} \Phi_{v}(\omega) \cdot\left[\begin{array}{cc}
\Phi_{u}(\omega) & \Phi_{e u}(\omega) \\
\Phi_{u e}(\omega) & \lambda_{0}
\end{array}\right]^{-1} .
$$

With the relation $\Phi_{u e}=-C S_{0} H_{0} \lambda_{0}$ and using the fact that $\Phi_{u} \lambda_{0}-\left|\Phi_{u e}\right|^{2}=\lambda_{0} \Phi_{u}^{r}$ it follows that

$$
\operatorname{cov}\left(\begin{array}{c}
\hat{G} \\
\hat{H}
\end{array}\right) \sim \frac{n}{N} \frac{\Phi_{v}}{\Phi_{u}^{r}} \cdot\left[\begin{array}{cc}
1 & \left(C S_{0} H_{0}\right)^{*} \\
C S_{0} H_{0} & \frac{\Phi_{u}}{\lambda_{0}}
\end{array}\right] .
$$

The variance expressions for $\hat{G}$ and $\hat{H}$ then become:

$$
\begin{aligned}
& \operatorname{cov}(\hat{G}) \sim \frac{n}{N} \frac{\Phi_{v}}{\Phi_{u}^{r}}=\frac{n}{N} \frac{\Phi_{v}}{\Phi_{u}}\left[1+\frac{\Phi_{u}^{e}}{\Phi_{u}^{r}}\right] \\
& \operatorname{cov}(\hat{H}) \sim \frac{n}{N} \frac{\Phi_{v}}{\lambda_{0}} \frac{\Phi_{u}}{\Phi_{u}^{r}}=\frac{n}{N} \frac{\Phi_{v}}{\lambda_{0}}\left[1+\frac{\Phi_{u}^{e}}{\Phi_{u}^{r}}\right] .
\end{aligned}
$$

The case of an open-loop experimental situation now appears as a special situation in which $\Phi_{u}^{e}=0, \Phi_{u}^{r}=$ $\Phi_{u}$, and $C=0$, leading to the well known expressions

$$
\operatorname{cov}(\hat{G}) \sim \frac{n}{N} \frac{\Phi_{v}}{\Phi_{u}} \quad \operatorname{cov}(\hat{H}) \sim \frac{n}{N} \frac{\Phi_{v}}{\lambda_{0}} .
$$

As indicated in Ljung (1993), the closed-loop expressions show that only the noisefree part $u^{r}$ of the input signal contributes to variance reduction of the estimates. The given expressions are restricted to the situation that $\mathcal{S} \in \mathcal{M}$ and that both $G(\theta)$ and $H(\theta)$ are identified.

Remark 3.1. The situation of estimating a plant model in the situation $G_{0} \in \mathcal{G}$ and having a fixed and correct noise model $H_{*}=H_{0}$ is considered in Ljung (1993) and is shown to be given by $\operatorname{cov}(\hat{G}) \sim \frac{n}{N} \frac{\Phi_{v}}{\Phi_{u}}$. This is a smaller variance than the situation in which both $G$ and $H$ are estimated.

\section{INDIRECT IDENTIFICATION}

\subsection{Introduction}

Recently several different indirect approaches to closedloop identification have been presented, see e.g. Gevers (1993) and Van den Hof and Schrama (1995). These methods have been introduced from considerations related to the bias that occurs in closed-loop identification of approximate models. Here we will briefly illustrate their properties with respect to the variance of the estimates.

\subsection{Coprime factor identification}

Coprime factor identification is treated in detail in Van den Hof et al. (1995). It is a scheme that relates to (and 
generalizes) the classical joint input/output method of closed-loop identification as e.g. described in Gustavsson et al. (1977). It does not require knowledge of the implemented controller $C$.

The basic principle is that the (two-times-two) transfer function $(r, e)^{T} \rightarrow(y, u)^{T}$ is identified, while the plant models $(\hat{G}, \hat{H})$ are retrieved from these closed-loop estimates. Consider the system's relations, using a filtered signal $x(t):=F(q) r(t)$ :

$$
\begin{aligned}
& y(t)=N_{0, F} x(t)+S_{0} H_{0} e(t) \\
& u(t)=D_{0, F} x(t)-C S_{0} H_{0} e(t)
\end{aligned}
$$

with $N_{0, F}:=G_{0} S_{0} F^{-1}$ and $D_{0, F}:=S_{0} F^{-1}$, constituting a coprime factor representation of $G_{0}$ as $G_{0}=$ $N_{0, F} D_{0, F}^{-1}$.

The linear and stable filter $F$ can be chosen by the user to serve several purposes, like minimal order properties or normalization of the coprime factorization as discussed in Van den Hof et al. (1995); this will not be pursued here any further as it is immaterial for the variance analysis. The important observation here is that the signals $x$ and $e$ are uncorrelated. Identification of the 4 transfer functions in (6),(7) from the signals $x(t), y(t)$, $u(t)$ therefore corresponds to a one-input two-output open-loop identification problem. Denote

$$
\begin{aligned}
& \varepsilon_{y}(t, \theta)=W_{y}(q, \theta)^{-1}[y(t)-N(q, \theta) x(t)] \\
& \varepsilon_{u}(t, \theta)=W_{u}(q, \theta)^{-1}[y(t)-D(q, \theta) x(t)]
\end{aligned}
$$

Least squares minimization of $\left(\varepsilon_{y}, \varepsilon_{u}\right)^{T}$ provides estimated models $\hat{N}, \hat{D}, \hat{W}_{y}, \hat{W}_{u}$.

Open-loop models $\hat{G}$ and $\hat{H}$ are then retrieved by

$$
\begin{aligned}
\hat{G} & =\hat{N}(\hat{D})^{-1} \\
\hat{H} & =(1+C \hat{G}) \hat{W}_{y} .
\end{aligned}
$$

For the variance of $\hat{G}$ and $\hat{H}$, use can be made of first order approximations: $\hat{G}=G_{0}+\Delta G, \hat{N}=N_{0, F}+\Delta N$, $\hat{D}=D_{0, F}+\Delta D$ etcetera, leading to

$$
\begin{aligned}
\Delta G & =\frac{\Delta N}{D_{0, F}}-\frac{N_{0, F} \Delta D}{D_{0, F}^{2}} \\
\Delta H & =\left(1+C G_{0}\right) \Delta W_{y}+C(\Delta G) W_{y} .
\end{aligned}
$$

This leads to the result:

$$
\operatorname{cov}\left(\begin{array}{c}
\hat{G} \\
\hat{H}
\end{array}\right) \sim \frac{n}{N} \frac{\Phi_{v}}{\Phi_{u}^{r}} \cdot\left[\begin{array}{cc}
1 & \left(C S_{0} H_{0}\right)^{*} \\
C S_{0} H_{0} & \frac{\Phi_{u}}{\lambda_{0}}
\end{array}\right]
$$

A sketch of the derivation of this result is given in the Appendix. Note that (9) is identical to expression (3) for direct identification .

\subsection{Identification in a dual Youla-Kucera parametrization}

Another method that has recently been introduced utilizes a specific parametrization of the plant $G_{0}$. As $C$ stabilizes the plant, $G_{0}$ can be parametrized within the class of all plants that are stabilized by $C$. This parametrization involves the relation

$$
G(\theta)=\frac{N_{x}+D_{c} R(\theta)}{D_{x}-N_{c} R(\theta)}
$$

where $N_{x} / D_{x}=: G_{x}$ is any (auxiliary) system that is stabilized by $C ; N_{c} / D_{c}=C$, and $R(\theta)$ ranges over the class of all stable proper transfer functions. The different factors that build up the quotient expressions $G_{x}$ and $C$ are required to be stable and coprime.

Using an expression like (10) for the plant $G_{0}$ with a Youla-Kucera parameter $R_{0}$, and substituting this in the system's relations, shows -after some manipulationsthat these can be rewritten as

$$
z(t)=R_{0} x(t)+W_{0} e(t)
$$

with $R_{0}=D_{x} S_{0}\left(G_{0}-G_{x}\right) / D_{c}, W_{0}=H_{0} S_{0} / D_{c}$, and

$$
\begin{aligned}
& z=\left(D_{c}+G_{x} N_{c}\right)^{-1}\left(y-G_{x} u\right) \\
& x=\left(D_{x}+C N_{x}\right)^{-1} r .
\end{aligned}
$$

Since $x$ is not correlated with $e$, the identification of $R_{0}$ and $W_{0}$ can again be considered as an open-loop identification problem. The signals $z$ and $x$ can be constructed by the user, as they are dependent on known quantities and measured signals. Least-squares identification is performed on the basis of the prediction error

$$
\varepsilon_{z}(t, \theta)=W(q, \theta)^{-1}[z(t)-R(q, \theta) x(t)]
$$

and the estimated transfers are denoted by $\hat{W}$ and $\hat{R}$. The open-loop model can then be reconstructed from these estimates according to

$$
\begin{aligned}
\hat{G} & =\frac{N_{x}+D_{c} \hat{R}}{D_{x}-N_{c} \hat{R}} \\
\hat{H} & =\hat{W} D_{c} \hat{S}^{-1}=\hat{W} D_{c}[1+C \hat{G}] .
\end{aligned}
$$

In order to guarantee that $\hat{H}$ is monic it will assumed that $D_{c}$ is monic.

Variance expressions for $\hat{R}$ and $\hat{W}$ are available through the standard (open-loop) expressions:

$$
\operatorname{cov}(\hat{R}) \sim \frac{n}{N} \frac{\left|W_{0}\right|^{2} \lambda_{0}}{\Phi_{x}} \text { and } \operatorname{cov}(\hat{W}) \sim \frac{n}{N}\left|W_{0}\right|^{2}
$$

while $\operatorname{cov}(\hat{R}, \hat{W})=0$. In a similar way as in section 4.2 , the variance of $(\hat{G}, \hat{H})$ can be obtained, relying on first order approximating expressions. Not surprisingly (see Appendix) the resulting expressions are again given by (9). 
Further details on this identification method can be found in and Van den Hof and Schrama (1995). It can be shown that it is a direct generalization of the classical indirect method of closed-loop identification, see Van den Hof and De Callafon (1996).

\subsection{Two-stage method}

A two-stage method for closed-loop identification has been introduced in Van den Hof and Schrama (1993). It operates directly on reference, input and output data, and does not require knowledge of the implemented controller. It can best be explained by considering the system's relations:

$$
\begin{aligned}
& y(t)=G_{0} u^{r}(t)+S_{0} H_{0} e(t) \\
& u(t)=S_{0} r(t)-C S_{0} H_{0} e(t) .
\end{aligned}
$$

In the first step, measured signals $r$ and $u$ are used to estimate a model $\hat{S}$ of the sensitivity function $S_{0}$. Next this model is used to construct (by simulation) an estimate $\hat{u}^{r}$ of $u^{r}$ according to $\hat{u}^{r}(t)=\hat{S}(q) r(t)$. In the second stage, the signals $\hat{u}^{r}$ and $y$ are used as a basis for the identification of a plant model $\hat{G}$.

The procedure is very much alike the coprime factor identification scheme, albeit that the final plant model is not calculated through division of two identified models; this division is circumvented by constructing the auxiliary simulated signal $\hat{u}^{r}=S(q, \hat{\gamma}) r$.

Consider the prediction errors

$$
\begin{aligned}
\varepsilon_{y}(t, \theta, \gamma) & =W_{y}^{-1}[y(t)-G(q, \theta) S(q, \gamma) r(t)] \\
\varepsilon_{u}(t, \gamma) & =W_{u}^{-1}[u(t)-S(q, \gamma) r(t)]
\end{aligned}
$$

then the parameter estimate $\hat{\theta}_{N}$ of this method can be written as the minimizing argument of $V_{N}(\lambda)$ for $\lambda \rightarrow$ $\infty$, with

$$
V_{N}(\lambda)=\frac{1}{N} \sum_{t=1}^{N}\left[\frac{1}{\lambda} \varepsilon_{y}^{2}(t, \theta, \gamma)+\cdot \varepsilon_{u}^{2}(t, \gamma)\right]
$$

(Note that for $\lambda \rightarrow \infty, \hat{\gamma}$ will be determined fully on the basis of $r$ and $u$ ). Applying the coprime factor results from section 4.2 to this situation then shows that the variance results are equivalent, and independent of $\lambda .{ }^{3}$

\subsection{Summarizing comments}

For the considered indirect methods, the asymptotic variance expressions for plant and noise model are exactly the same as the expressions for direct identification. This may not be too surprising, as similar results

3 The authors acknowledge the contribution of Urban Forssell (Univ. Linköping) to the proof of this result. for the classical indirect and joint i/o methods were already available (Gustavsson et al., 1977). However what has to be stressed here, is that for the indirect type methods the variance expressions for $\hat{G}$ are valid also in the situation that $G_{0} \in \mathcal{G}$ but $\mathcal{S} \notin \mathcal{M}$, while for the direct method the results are only achieved under the stronger condition that $\mathcal{S} \in \mathcal{M}$.

\section{OPEN-LOOP VERSUS CLOSED-LOOP EXPERIMENTS}

Considering that the variance expressions are identical for all closed-loop identification methods, we can now make a comparison between the variances obtained from open-loop and closed-loop experimental conditions. The appropriate expressions are summarized in table 1 .

\begin{tabular}{c|ccc} 
& Open-loop & Closed-loop \\
\hline $\operatorname{Var}\left(\hat{G}_{N}\right)$ & $\frac{n}{N} \frac{\Phi_{v}}{\Phi_{u}}$ & $<$ & $\frac{n}{N} \frac{\Phi_{v}}{\Phi_{u}^{r}}$ \\
$\operatorname{Var}\left(\hat{H}_{N}\right)$ & $\frac{n}{N} \frac{\Phi_{v}}{\lambda_{0}}$ & $<$ & $\frac{n}{N} \frac{\Phi_{v}}{\lambda_{0}}\left(1+\frac{\Phi_{u}^{e}}{\Phi_{u}^{r}}\right)$
\end{tabular}

Table 1. Variance expressions under openloop and closed-loop conditions.

The results show that for both $\hat{G}$ and $\hat{H}$ the variance obtained under closed-loop identification will generally be larger than for open-loop identification. Particularly in a situation where the input power is limited, the difference will become apparent, as in that case only part of the actual input spectrum can be used for variance reduction of $\hat{G}$ and $\hat{H}$. In case the input power is not restricted, closed-loop identification can achieve the same results as open-loop identification, by choosing a reference signal $r$ such that $\Phi_{u}^{r}$ is equal to the input spectrum applied in the open-loop situation.

The results suggest that in terms of variance of the model estimates $\hat{G}_{N}$ and $\hat{H}_{N}$, open-loop identification always has to be preferred over closed-loop identification. However, perhaps surprisingly, this is not the case if the objective of the identification is model-based control design, as is explained in the next section.

\section{OPTIMAL EXPERIMENTS IN VIEW OF MODEL-BASED CONTROL}

In this section we will consider the situation that the identified transfer functions $\hat{G}_{N}$ and $\hat{H}_{N}$ are used as a basis for model-based control design, and we will illustrate the effect of the variance of the identified model on the model application, i.e. the designed controller. To this end we will first consider the following result from Ljung (1987, Theorem 14.3). 
Proposition 6.1. Consider the variance-based identification design criterion

$$
J(\mathcal{D})=\int_{-\pi}^{\pi} \operatorname{tr}[P(\omega, \mathcal{D}) \Gamma(\omega)] d \omega
$$

where $P(\omega, \mathcal{D})=\operatorname{cov}\left[\hat{G}\left(e^{i \omega}\right) \hat{H}\left(e^{i \omega}\right)\right]^{T}, \mathcal{D}$ denotes the design choices with respect to the experimental conditions, represented by $\left\{\Phi_{u}, \Phi_{u e}\right\}$, while $\Gamma(\omega)$ is a $2 \times 2$ Hermitian matrix reflecting the intended application of the model.

If $\Gamma_{12}(\omega) \equiv 0$ and the input power is limited, then the experimental condition $\mathcal{D}$ for which $J(\mathcal{D})$ is optimized is given by

$$
\Phi_{u}^{o p t}=c \cdot \sqrt{\Gamma_{11}(\omega) \Phi_{v}(\omega)} \quad \Phi_{u e}^{o p t} \equiv 0
$$

and $c$ is a constant.

This result shows that open-loop identification is optimal when in the intended model application, the covariance between $\hat{G}$ and $\hat{H}$ is not penalized, but only the variance contributions of $\hat{G}$ and $\hat{H}$ separately. This situation applies e.g. to the case where a controller is designed on the basis of $\hat{G}$ only.

Corollary 6.2. Consider as model application a control design scheme based on a frequency weighted sensitivity minimization:

$$
C_{\hat{G}}=\arg \min _{\tilde{C}}\left\|V(1+\tilde{C} \hat{G})^{-1}\right\|_{2} .
$$

Then the optimal experiment design in line with the above proposition is given by

- open-loop experiments $\left(\Phi_{u e}^{o p t} \equiv 0\right)$.

- $\Phi_{u}^{o p t}=c \cdot\left|C_{\hat{G}} V S_{0}^{2}\right| \sqrt{\Phi_{v}}$

Proof. The application-related error criterion can be written as $\left\|V\left[\left(1+C G_{0}\right)^{-1}-(1+C \hat{G})^{-1}\right]\right\|_{2}$ which can be shown to be equal to (using first order approximations) $\left\|\frac{V C\left(G_{0}-\hat{G}\right)}{\left(1+C G_{0}\right)^{2}}\right\|_{2}$. An appropriate choice of $\Gamma_{11}$ for this model application would thus be $\Gamma_{11}(\omega)=\frac{|V C|^{2}}{\left|1+C G_{0}\right|^{4}}$ leading to the result presented.

From the above result one could conclude that -from a variance point of view- open-loop identification is optimal for this control design. However, the required input spectrum in this 'open-loop' situation should be proportional to the sensitivity function $S_{0}$ of the real plant, being controlled by the yet-to-be-designed controller. Input shaping with $S_{0}$ is exactly what is done in closedloop identification, since $\Phi_{u}=\left|S_{0}\right|^{2} \Phi_{r}+\Phi_{u}^{e}$.

A second related result is present in the recent work of Hjalmarsson et al.(1996) on optimal identification for control. In this work the identification criterion is selected to minimize the control performance degradation that results from the random errors on $\hat{G}_{N}$ and $\hat{H}_{N}$. In solving this problem, the authors have quantified the variance error on the designed model-based controller.

Consider a situation where an identified model $\hat{G}_{N}, \hat{H}_{N}$ is obtained from a closed-loop experimental situation with a controller $C_{i d}$ implemented on the plant. Consider a model-based control design scheme

$$
\hat{C}_{N}=c\left(\hat{G}_{N}, \hat{H}_{N}\right)
$$

and let $F_{G}, F_{H}$ reflect the derivatives of $c$ with respect to $G, H$, i.e. the sensitivity of the controller with respect to changes in $G$ and $H$. Then the variance of the controller estimate is (see Hjalmarsson et al., 1996)

$$
\begin{aligned}
& \operatorname{cov}\left(\hat{C}_{N}\right) \sim \frac{n}{N}\left|H_{0}\right|^{2} . \\
& \left\{\left|F_{H}\right|^{2}+\frac{\lambda_{0}}{\Phi_{r}}\left|F_{G}+\left(F_{G} G_{0}+F_{H} H_{0}\right) C_{i d}\right|^{2}\right\}
\end{aligned}
$$

leading to the following situations.

Situation $F_{H} \neq 0$. The controller variance is minimized for models identified in closed-loop with an implemented controller $C_{i d}^{o p t}$ unequal to zero, and the resulting controller variance is

$$
\operatorname{cov}\left(\hat{C}_{N}\right) \sim \frac{n}{N}\left|H_{0}\right|^{2}\left|F_{H}\right|^{2}
$$

By comparison, the controller variance obtained with open-loop identification is

$$
\operatorname{cov}\left(\hat{C}_{N}\right) \sim \frac{n}{N}\left|H_{0}\right|^{2}\left|F_{H}\right|^{2}\left(1+\frac{\left|F_{G}\right|^{2}}{\left|F_{H}\right|^{2}} \cdot \frac{\lambda_{0}}{\Phi_{u}}\right) .
$$

We observe that the variance obtained under ideal closedloop experimental conditions can only be achieved with open-loop identification if the input power is made infinite.

Situation $F_{H}=0$. The variance expression becomes

$$
\operatorname{cov}\left(\hat{C}_{N}\right) \sim \frac{n}{N}\left|H_{0}\right|^{2}\left|F_{G}\right|^{2} \frac{\left|1+C_{i d} G_{0}\right|^{2} \lambda_{0}}{\Phi_{r}}=\frac{n}{N} \frac{\Phi_{v}}{\Phi_{u}^{r}}\left|F_{G}\right|^{2} .
$$

The corresponding expression for open-loop identification is

$$
\operatorname{cov}\left(\hat{C}_{N}\right) \sim \frac{n}{N} \frac{\Phi_{v}}{\Phi_{u}}\left|F_{G}\right|^{2}
$$

The situation $F_{H}=0$ means that the control design depends only on $G$ and not on the noise model. This result is therefore consistent with Corollary 6.2.

We conclude from this analysis that, as far as variance errors are concerned, for model-based control design, closed-loop identification is optimal except when the controller is independent of the noise model. 


\section{CONCLUSIONS}

Asymptotic variance expressions have been derived for several closed-loop identification schemes, involving both the (classical) direct method and more recently introduced indirect identification methods. It is shown that the several approaches lead to the same asymptotic variance. Although asymptotic variance of plant model and noise model generally will increase when performing closed-loop identification, in comparison with open-loop identification, closed-loop identification can still be preferred when the identified model is used as a basis for control design. In the case that a controller is designed on the basis of both plant model and noise model, closedloop identification is shown to lead to better variance results.

\section{APPENDIX}

\section{Proof of (9).}

Applying the standard variance expressions to the multivariable situation of $(6),(7)$ it follows that

$$
\begin{aligned}
\operatorname{cov}\left(\begin{array}{c}
\hat{N} \\
\hat{D}
\end{array}\right) \sim \frac{n}{N} \frac{\left|S_{0}\right|^{2} \Phi_{v}}{\Phi_{x}}\left[\begin{array}{cc}
1 & -C^{*} \\
-C & |C|^{2}
\end{array}\right] \\
\operatorname{cov}\left(\begin{array}{c}
\hat{W}_{y} \\
\hat{W}_{u}
\end{array}\right) \sim \frac{n}{N} \frac{\left|S_{0}\right|^{2} \Phi_{v}}{\lambda_{0}}\left[\begin{array}{cc}
1 & -C^{*} \\
-C & |C|^{2}
\end{array}\right] .
\end{aligned}
$$

Since (6),(7) reflect an open-loop situation (as $x$ and $e$ are uncorrelated) this implies that the cross-covariance terms between $(\hat{N}, \hat{D})^{T}$ and $\left(\hat{W}_{y}, \hat{W}_{u}\right)$ are zero. From the first order approximations in (8) it follows that $|\Delta G|^{2}=$

$$
\frac{|\Delta N|^{2}}{\left|D_{0, F}\right|^{2}}+\frac{\left|G_{0}\right|^{2}}{\left|D_{0, F}\right|^{2}}|\Delta D|^{2}-2 \operatorname{Re}\left\{\frac{G_{0}(\Delta D)(\Delta N)^{*}}{\left|D_{0, F}\right|^{2}}\right\} .
$$

Substitution of (A.1) then provides the result for $\operatorname{cov}(\hat{G})$. For $\hat{H}$ one can similarly write (when neglecting terms that have expectation 0 ):

$$
|\Delta H|^{2}=\left|1+C G_{0}\right|^{2}\left|\Delta W_{y}\right|^{2}+\left|C W_{y}\right|^{2}|\Delta G|^{2}
$$

and the result for $\operatorname{cov}(\hat{H})$ follows after substitution of (A.2). The expression for $\operatorname{cov}(\hat{G}, \hat{H})$ follows from $\operatorname{cov}(\hat{G}, \hat{H})=-\left(C W_{y}\right)^{*} \operatorname{cov}(\hat{G})$.

\section{Variance result for dual Youla-Kucera method}

Using (11),(12) the related expressions for the first order approximation errors become

$$
\begin{gathered}
\Delta G=\frac{\left(D_{x}-N_{c} R_{0}\right) D_{c}(\Delta R)+\left(N_{x}+D_{c} R_{0}\right) N_{c}(\Delta R)}{\left(D_{x}-N_{c} R_{0}\right)^{2}} \\
\Delta H=\frac{D_{c}(\Delta W)}{S_{0}}+W_{0} N_{c}(\Delta G) .
\end{gathered}
$$

For $\Delta G$ this leads to

$$
\Delta G=\frac{D_{c}+G_{0} N_{c}}{D_{x}-N_{c} R_{0}} \Delta R=\frac{D_{c}(\Delta R)}{D_{x} S_{0}^{2}\left(1+C G_{x}\right)}
$$

and so

$$
\operatorname{cov}(\hat{G})=\left|\frac{D_{c}}{D_{x} S_{0}^{2}\left(1+C G_{x}\right)}\right|^{2} \operatorname{cov}(\hat{R}) .
$$

Substituting the expression for $\operatorname{cov}(\hat{R})$ and using the property that $\Phi_{x}=\left|D_{x}\left(1+C G_{x}\right)\right|^{2} \Phi_{r}$ it follows after some manipulation that $\operatorname{cov}(\hat{G}) \sim n / N \cdot \Phi_{v} / \Phi_{u}^{r}$. For $\operatorname{cov}(\hat{H})$ it follows from (A.4) that

$$
\operatorname{cov}(\hat{H})=\frac{\left|D_{c}\right|^{2} \operatorname{cov} \hat{W}}{\left|S_{0}\right|^{2}}+\left|N_{c} W_{0}\right|^{2} \operatorname{cov} \hat{G} .
$$

Substituting the known expressions in the right hand side, will show that $\operatorname{cov}(\hat{H}) \sim n / N\left|H_{0}\right|^{2}\left[1+\Phi_{u}^{e} / \Phi_{u}^{r}\right]$. For $\operatorname{cov}(\hat{G}, \hat{H})$ it follows from (A.4) that $\operatorname{cov}(\hat{G}, \hat{H})=$ $\left(W_{0} N_{c}\right)^{*} \operatorname{cov}(\hat{G})$ which leads to the appropriate result.

\section{REFERENCES}

Gevers, M. (1993). Towards a joint design of identification and control? In: H.L. Trentelman and J.C. Willems (Eds.), Essays on Control: Perspectives in the Theory and its Applications. Birkhäuser, Boston, pp. 111-151.

Gustavsson I., L. Ljung and T. Söderström (1977). Identification of processes in closed loop - identifiability and accuracy aspects. Automatica, 13, 59-75.

Hansen, F.R. and G.F. Franklin (1988). On a fractional representation approach to closed-loop experiment design. Proc. American Control Conf., Atlanta, GA, USA, pp. 1319-1320.

Hjalmarsson, H., M. Gevers and F. De Bruyne (1996). For model-based control design, closed-loop identification gives better performance. Automatica, 32, 16591673.

Ljung, L. (1987). System Identification: Theory for the User. Prentice-Hall, Englewood Cliffs, NJ.

Ljung, L. (1993). Information contents in identification data from closed-loop operation. Proc. 32nd IEEE Conf. Dec. Contr., San Antonio, TX, pp. 2248-2252.

Van den Hof, P.M.J., R.J.P. Schrama, R.A. de Callafon and O.H. Bosgra (1995). Identification of normalised coprime plant factors from closed-loop experimental data. Europ. J. Control, 1, 62-74.

Van den Hof, P.M.J. and R.J.P. Schrama (1995). Identification for control - closed-loop issues. Automatica, 31, 1751-1770.

Van den Hof, P.M.J. and R.A. de Callafon (1996). Multivariable closed-loop identification: from indirect identification to dual-Youla parametrization. Proc. 35th IEEE Conf. Dec. Contr., Kobe, Japan, pp. 1397-1402. 